\title{
Recent clinical data regarding the use of $\beta$ blockers in heart failure: focus on CIBIS II
}

\author{
H Dargie
}

From studies which first started in Sweden many years ago, the evidence for the beneficial role of $\beta$ blockers in the management of heart failure is now very substantial. We have considerable data from the meta-analyses of many small trials and from the second cardiac insufficiency bisoprolol study (CIBIS II). ${ }^{1-3}$ The modern era of large randomised control trials involving $\beta$ blockers really started with the US carvedilol heart failure study, in which a very substantial reduction in mortality (which was not the primary aim of the study programme) and morbidity was shown with carvedilol. ${ }^{4}$ Another study with carvedilol was the smaller Australia-New Zealand heart failure research group trial of around 300 patients following myocardial infarction. ${ }^{5}$ This showed a reduction in a composite end point of hospitalisations but focused on one aspect - the effect on left ventricular function-which suggested that it was improved by carvedilol. This effect has now become an important feature of $\beta$ blocker treatment.

\section{CIBIS II trial}

The CIBIS II trial was based on the first CIBIS study, which showed a non-significant $20 \%$ reduction in mortality but a significant reduction in hospital admissions as a result of worsening heart failure. ${ }^{67}$ CIBIS II was a much larger trial, comprising a total of 2647 patients. It was the first of the very large trials with sufficient power to address all cause mortality as the primary end point. The trial was stopped prematurely because there was a highly significant difference in mortality in favour of bisoprolol.

The primary end point in this trial was all cause mortality, while the secondary end points were cardiovascular mortality, hospital admissions, and permanent withdrawal from treatment

The patients who went into the CIBIS II trial were stable and receiving angiotensin converting enzyme (ACE) inhibitors and diuretics. They were not hospital inpatients but were recruited from the outpatient clinic. The criteria were that patients should be stable with no change of treatment for a minimum period of two weeks. The trial inclusion criteria may prove helpful in defining who might be eligible for $\beta$ blocker treatment. Certainly patients admitted for stabilising would not be deemed suitable. Recruitment was extended up to the age of 80 years but there were also some very young patients. All patients were required to have left ventricular systolic dysfunction.

There were not very many exclusion criteria. Disallowed concomitant medications were $\beta$ blockers, calcium channel blockers, and ino- tropes, with the exception of digitalis. Amiodarone was permitted on the grounds that there are now a large number of trials of amiodarone in heart failure which suggest benefit. ${ }^{8}$ It is postulated that benefit is derived from concomitant $\beta$ blocker treatment. ${ }^{9}$

PATIENT CHARACTERISTICS

Patients were relatively young, with an average age of 61 years, and mostly male. The great majority had New York Heart Association (NYHA) class III heart failure with a substantially reduced ejection fraction of $\leqslant 35 \%$. Ischaemic heart disease was by far the most common shared characteristic. To have documented ischaemic heart disease, it was necessary to prove evidence of myocardial infarction by common definition, or to have undergone coronary angiography. To have documented dilated cardiomyopathy, left ventricular systolic dysfunction had to be evident but with normal coronary arteries at angiography.

The percentage of patients recruited to CIBIS II with dilated cardiomyopathy (12\%) was relatively small in comparison to the first CIBIS trial, where the apparent rate of cardiomyopathy was nearly a third. In the earlier trial, however, coronary angiography had not been a prerequisite for a diagnosis of cardiomyopathy and a number of patients remained undefined; they probably had ischaemic heart disease but their condition had not been investigated.

CIBIS II was a European trial with the greatest percentage of patients (30\%) coming from Russia, and a further significant proportion originating from Poland (11.6\%). There were concerns expressed regarding whether these patients were typical of those from western Europe as a whole. However, it appeared all patients were receiving ACE inhibitors so there were no reasons to believe they were treated differently from elsewhere in Europe. Although standard treatment was one of the inclusion criteria, eastern European patients had not been prescribed drugs solely for the purposes of entry to CIBIS II but had been on them for some time. The dosages of captopril and enalapril were relatively small but were typical of dosages commonly prescribed in both western and eastern Europe. The most common dose of bisoprolol during titration and the follow up phase was $10 \mathrm{mg} ; 50-60 \%$ of patients went on to the highest dose and only relatively small numbers of patients received the lower doses (range 1.25-10 mg once daily).

\section{RESULTS}

A substantial reduction in all cause mortality was seen in the bisoprolol treated patients. Mortality was $17.3 \%$ in patients receiving 
Table 1 CIBIS II: primary end points

\begin{tabular}{lllll}
\hline Primary end point & $\begin{array}{l}\text { Placebo } \\
(n=1320)\end{array}$ & $\begin{array}{l}\text { Bisoprolol } \\
(n=1327)\end{array}$ & $\begin{array}{l}\text { Hazard ratio } \\
(95 \% \text { CI })\end{array}$ & $p$ Value \\
\hline All cause mortality & $228(17 \%)$ & $156(12 \%)$ & $0.66(0.54$ to 0.81$)$ & $<0.0001$
\end{tabular}

Numbers refer to patients who presented at least once with given events.

CI, confidence interval.

Reproduced from reference 7, with permission of The Lancet Ltd.

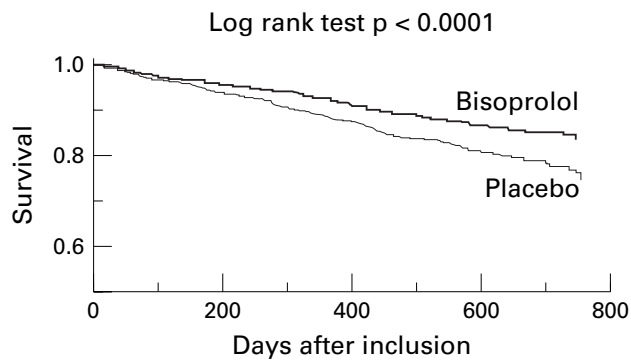

Figure 1 Survival curves from CIBIS II: all cause mortality. Annual mortality - bisoprolol $8.8 \%$, placebo $13.2 \%$; average follow up 1.3 years. Reproduced from reference 7, with permission of The Lancet Ltd.

placebo compared with $11.8 \%$ in the bisoprolol treated patients (table 1). Looking at the survival curves, this translates into an annual mortality rate of $13.2 \%$ in the placebo group versus $8.8 \%$ in the bisoprolol group over an average follow up of 1.3 years $(\mathrm{p}<0.0001)$ (fig $1)$. The NYHA class in these patients was intended to be III and IV. While mortality was reasonably high among this group of patients, the number of deaths was not as high as might have been expected for patients in class III and IV. Of the secondary end points, there was a substantial reduction in cardiovascular mortality (table 2). Hospital admissions were reduced by $20 \%$ in the bisoprolol treated patients compared with placebo, while premature treatment withdrawals were the same for both groups of patients-about $15 \%$ of the total-so treatment appeared to be reasonably well tolerated.

With regard to the causes of death, sudden deaths (defined as death within an hour without preceding symptoms) were very significantly reduced by $44 \%(\mathrm{p}=0.001)$. There was also a trend to reduction in pump failure deaths, a condition which was very strictly defined by the critical end points committee. Various other causes of death were classified, including unknown causes (although these were almost certainly also cardiovascular) which were more than halved in the bisoprolol treated group.

Hospital admissions were reduced for all causes but the $36 \%$ reduction in admissions for heart failure was particularly striking. There

Table 2 CIBIS II: secondary end points

\begin{tabular}{lllll}
\hline Secondary end points & $\begin{array}{l}\text { Placebo } \\
(n=1320)\end{array}$ & $\begin{array}{l}\text { Bisoprolol } \\
(n=1327)\end{array}$ & $\begin{array}{l}\text { Hazard ratio } \\
(95 \% \text { CI })\end{array}$ & $p$ Value \\
\hline $\begin{array}{llll}\text { All cause hospital admissions } \\
\text { All cardiovascular deaths }\end{array}$ & $513(39 \%)$ & $440(33 \%)$ & $0.80(0.71$ to 0.91$)$ & 0.0006 \\
$\begin{array}{l}\text { Combined end point } \\
\begin{array}{l}\text { Permament treatment } \\
\text { withdrawals }\end{array}\end{array}$ & $161(12 \%)$ & $119(9 \%)$ & $0.71(0.56$ to 0.90$)$ & 0.0049 \\
& $192(15 \%)$ & $194(15 \%)$ & $1.00(0.82$ to 1.22$)$ & 0.98 \\
\hline
\end{tabular}

Numbers refer to patients who presented at least once with given events. For hospital admissions, numbers refer to patients admitted at least once with any cause.

Reproduced from reference 7, with permission of The Lancet Ltd. was also a notable reduction in important ventricular arrhythmias. Bradycardia was slightly more common in the bisoprolol treated patients, while hypotension (as a reason for admission) was more common in the patients on placebo.

One of the interesting findings was that hospitalisations for stroke appeared to occur less with placebo than with bisoprolol. No adequate explanation could be found to explain this finding, although patients on bisoprolol with stroke had more risk factors for stroke than patients on placebo. However the numbers are very small and it would be difficult to draw firm conclusions from them.

\section{MERIT-HF}

Another recent large $\beta$ blocker study is the metoprolol CR/XL randomised intervention trial in heart failure (MERIT-HF)..$^{11}$ This is a study of metoprolol in patients with depressed ejection fraction and symptoms of heart failure. Nearly 4000 patients were recruited from Europe and North America. The majority were recruited in Europe, again with many from eastern Europe, and $27 \%$ were recruited in the US. The age range of patients was similar to that of CIBIS II, comprising mostly men with NYHA class II and III heart failure and a small number of class IV patients. The average ejection fraction in MERIT patients, including a larger number of class II patients, was similar to CIBIS II. The heart failure of the majority had an ischaemic cause with about one third having a non-ischaemic origin.

There were 145 deaths in the metoprolol group and 217 in the placebo group, translating into a $34 \%$ risk reduction for $\beta$ blocker treatment, which started appearing fairly soon after recruitment, as was also found in CIBIS II. The $41 \%$ risk reduction for sudden death was also very similar. It is evident from observations of the proportion of sudden deaths that these occur more frequently where heart failure is least severe- that is, in patients with NYHA class II heart failure. This finding accords with epidemiological studies of mortality in patients with heart failure and is comparable to the CIBIS II data.

\section{Conclusions}

In summary, these trials tell us that $\beta$ blocker treatment in patients with $\mathrm{CHF}$ results in substantial reductions in mortality and improvements in left ventricular function. All cause mortality is reduced by around one third, in large part because of the reduction in sudden deaths. Death from heart failure in MERIT is probably halved by $\beta$ blocker treatment and there is a similar trend evident in the CIBIS II data. No data are as yet available from MERIT on hospitalisations but in CIBIS II hospitalisations were substantially reduced, particularly those for heart failure, which were reduced by over a third. Data from over 6000 patients have shown us that patients with NYHA class II, III, and IV heart failure, as defined in the protocol, tolerate $\beta$ blockers well.

Patients who were included in the trials were stable, attending the outpatient clinic, and were 
not on the ward being treated for heart failure. They were ambulatory and receiving standard heart failure treatment with loop diuretics and ACE inhibitors. Over half the patients in CIBIS II and MERIT were also taking digoxin. Patients studied so far have been relatively young; this was also the case in the ACE inhibitor trials of patients with systolic dysfunction. In a recent study at the Glasgow Western Infirmary, patients with heart failure admitted to the emergency system had an average age of 78 years. So although the typical age group is under represented in the data, there were some patients up to 80 years of age included in both CIBIS II and MERIT.

Whether we are justified in treating very elderly patients with $\beta$ blockers is, as yet, not absolutely clear. However, if patients are in other respects similar to those in CIBIS II, there is no apparent reason why they should not also be started on treatment, providing systems are in place to ensure safety.

The most robust results were seen in patients with moderate heart failure and in those with ischaemic heart disease. However, certainly in MERIT where there is a non-ischaemic cause, and also in CIBIS II where some of the causes were unknown, a large number of patients would also have ischaemic heart disease. Recent results show remarkable similarities.

$\beta$ BLOCKERS FOR CHF IN THE COMMUNITY

A different system is needed in order to implement $\beta$ blocker treatment for patients with moderate heart failure in the community. We would probably recommend that patients are initiated on treatment and followed up with at least some element of specialist supervision, and achieving this is going to be important. This does not necessarily mean that patients must always be under the care of specialists, but there needs to be an element of the specialist system in primary care. This may, for example, be provided by the use of liaison heart failure nurses to bridge the interface between hospital clinic and community.

Transferring trial results into clinical practice will require a re-evaluation of how we manage heart failure. It is important to complete the evidence based spectrum of benefit. For this we need more data from the elderly and from those with very severe forms of heart failure. This issue is being addressed in part by COPERNICUS (carvedilol prospective randomised cumulative survival trial), a study of carvedilol in patients with severe heart failure. ${ }^{12}$ There are also trials in progress involving patients with heart failure and left ventricular dysfunction after myocardial infarction.

The $\beta$ blocker evaluation survival trial (BEST) is the study of bucindolol in patients with heart failure. ${ }^{13}$ The entry criteria seem to be very similar to CIBIS II, in that the patients have NYHA class III and IV heart failure. However, this trial has recently been stopped because of an absence of significant survival advantage in the bucindolol treatment group. The issue of treating patients in the community is important because there are many with the substrates for heart failure who might also respond well to $\beta$ blocker treatment. However, we cannot yet assume this and the area needs to be researched further before treatment is widely used.

1 Swedburg K. History of beta blockers in congestive heart failure. Heart 1998;79(suppl 2): S29-30.

2 Lechat P, Jaillon P, Fontaine ML, et al. A randomised trial of beta blockade in heart failure: the cardiac insufficiency bisoprolol study (CIBIS). Circulation 1994;90:1765-73.

3 Waagstein F, Bristow MR, Swedburg K, et al. Beneficial effects of metoprolol in idiopathic dilated cardiomyopathy. Lancet 1993; 342:1441-6.

4 Packer M, Bristow MR, Cohn JN, et al. The effect of carvedilol on morbidity and mortality in patients with chronic heart failure. N Engl f Med 1996;334:1349-55.

5 MacMahon S, Sharpe N, Doughty R, et al. Randomised, placebo-controlled trial of carvedilol in patients with congestive heart failure due to ischaemic heart disease. Lancet 1997;349:375-80.

6 The CIBIS II Scientific Committee. Design of the cardiac insufficiency bisoprolol study II (CIBIS II). Fundam Clin Pharmacol 1997;11:138-42.

7 The cardiac insufficiency bisoprolol study II (CIBIS II): a randomised trial. Lancet 1999;353:9-13.

8 Boutitie F, Boisssel JP, Connolly SJ, et al. Amiodarone interaction with beta blockers: analysis of the merged EMIAT (European myocardial infarct amiodarone trial) and CAMIAT (Canadian amiodarone myocardial infarction trial) databases. Circulation 1999;99:2268-75.

9 Piepoli M, Villani GQ, Ponikowski P, et al. Overview and meta analysis of randomised trials of amiodarone in chronic heart failure. Int $\mathcal{F}$ Cardiol 1998;66:1-10.

10 Hjalmarson A, Goldstein S, Fagerberg B, et al. Rationale, design and organisation of the metoprolol CR/XL randomised intervention trial in heart failure (MERIT-HF). Am f Cardiol 1997;80:J54-8.

11 Hjalmarson A, Goldstein S, Fagerberg B, et al. Effect of metoprolol $\mathrm{CR} / \mathrm{XL}$ in chronic heart failure: metoprolol $\mathrm{CR} / \mathrm{XL}$ randomised intervention trial in congestive heart failure (MERIT-HF). Lancet 1999;353:2001-7.

12 Packer M. Effects of beta-adrenergic blockade on survival in patients with chronic heart failure. Am 7 Cardiol 1997;80 (suppl):46-54L

13 BEST Steering Committee. Design of the beta-blocker evaluation survival trial (BEST). Am f Cardiol 1995;75: 1220-3. 